\title{
openEHR Based Contextual Problem List
}

\author{
John MEREDITH ${ }^{\mathrm{a}, 1}$, Ian MCNICOLL ${ }^{\mathrm{b}}$, Nik WHITEHEAD ${ }^{\mathrm{c}}$ and Michael DACEY \\ ${ }^{a}$ Wales Institute for Digital Information, Cardiff UK \\ ${ }^{\mathrm{b}}$ OpenEHR International, London UK \\ ${ }^{\mathrm{c}}$ University of Wales Trinity Saint David, Swansea UK
}

\begin{abstract}
The problem list is a key facet of the digital patient record that has historically been difficult to curate. This paper presents an implementation of a contextual problem list using openEHR. It describes the modelling approach, key model elements, and how these are assembled to underpin a Problem Oriented Medical Record. Finally, it discusses issues associated with how problem lists may be used.
\end{abstract}

Keywords. Contextual problem list, problem oriented medical record, clinical models, diagnosis, openEHR, digital health.

\section{Introduction}

The contextual problem list (CPL, [1]) presents a hierarchy of conditions and diagnoses for a range of clinical disciplines, split into categories such as primary diagnosis or co-morbidity. The model is based upon the Problem Oriented Medical Record (POMR) as pioneered by Dr Lawrence Weed[2]. An openEHR[3] implementation of the CPL can be used to address some of the criticisms levelled at POMR[4], facilitating records to exist "across problem boundaries". The key attributes of the CPL as applied via an openEHR archetype-based model are described here.

\section{Methods}

The published CPL template[5] comprises of a Problem/Diagnosis archetype to capture the problem itself, and a Problem/diagnosis qualifier to add context-specific elements such as problem status or its resolution. Problem categories (i.e. primary, comorbidity or complication) are characterised by discrete sections within the overall template. To support the CPL for multiple care pathways, additional metadata elements are required to capture the clinical context. The template can facilitate an unambiguous absence of information statement where a given CPL category has "No known information". This is the initial state of each CPL category. By utilising openEHR architecture, each CPL can contribute to the master problem list (MPL), akin to the longitudinal list originally envisaged by Weed[2]. This can be derived from the contents of multiple CPLs as each problem entry is represented as an individual record.

\footnotetext{
${ }^{1}$ John Meredith, Wales Institute for Digital Information, 21 Cowbridge Road East, Cardiff CF11 9AD, UK; E-mail: john.meredith@wales.nhs.uk.
} 


\section{Results and Discussion}

By utilising a digital composition derived from an openEHR based system, it is possible to both view a problem list with a specific clinical perspective such as Diabetes, and a master list of all coded entries. This master list is core component of the EHR, as described by the Institute of Medicine [6]. We suggest that the ownership of each problem list by a clinical specialty will address the historical issue of completeness[4]. This in turn increases accuracy and completeness of the master problem list. Realisation is heavily dependent on the implementation of a reconciliation process to ensure new records are presented in a timely, usable manner.

Reconciliation of medication charts is common where patients move between care domains such as primary care to acute[7]. This concept, when applied to CPL is vital to ensure that up-to-date lists are aligned with any clinical pathway being used to manage the patient's care. Ownership of the list reduces the overall burden of curation as it directly links to individual episodes of care. A responsible clinician will be concerned with the management of problems at a point in time, with each CPL instantiation contributing to the overall longitudinal perspective. This may also present an opportunity to apply business rules and clinical decision support with a view to preventative medicine.

However, the reconciliation of problem records may present privacy or safety concerns for potentially sensitive entries (e.g. those recorded for sexual health). As a result, business logic to exclude or restrict use may be required. Additionally, there is the danger that similar, related entries may be present on multiple problem lists which leads to duplication. The hierarchal nature of SNOMED CT may facilitate grouping of related terms to ease the reconciliation process, whilst still giving the user data entry autonomy.

\section{Conclusion}

This paper describes how the CPL can be implemented into openEHR based systems as a standardised component. It facilitates the view of patient problems for a specific clinical specialty or across several care domains, maintaining the semantic context from which a given CPL can be viewed. This feasibility in turn makes timely reconciliation of new data viable, without precluding longitudinal master problem list management.

\section{References}

[1] Meredith J, McNicoll I, Whitehead N, Ademoye K. Defining the Contextual Problem List. Stud Health Technol Inform. 2020 Jun 16;270:567-71.

[2] Weed LL. Medical Records That Guide and Teach. N Engl J Med. 1968 Mar 14;278(11):593-6

[3] openEHR Foundation. What is openEHR? 2019. Available at: http://bit.ly/openehr, Accessed August 6, 2019.

[4] Galanter WL, Hier DB, Jao C, Sarne D. Computerized physician order entry of medications and clinical decision support can improve problem list documentation compliance. Int J Med Inform. 2010 May;79(5):332-8.

[5] Apperta UK. Contextual Problem List.v0, Draft template [Internet]. Apperta UK OpenEHR Clinical Knowledge Manager. 2019.

[6] Dick RS, Steen EB, Detmer DE, editors. The Computer-Based Patient Record. Washington, D.C.: National Academies Press; 1997; p. 256.

[7] Barnsteiner JH. Medication Reconciliation. In: Hughes R, editor. Patient safety and quality: an evidencebased handbook for nurses. Rockville (MD): Agency for Healthcare Research and Quality (US); 2008. 